\section{$\S 5$. Neoclassical Transport Optimization of LHD}

Murakami, S., Wakasa, A. (Hokkaido U.), Maassberg, H., Beidler, C.D. (Max-Planck-Institut fur Plasmaphysik, Germany)

The existence of the $1 / \mathrm{N}$ regime, in which the neoclassical transport coefficients are inversely proportional to the collision frequency, increases the neoclassical particle and heat transport in nonaxisymmetric devices to levels far above those of axisymmetric tokamaks in the fusion-relevant longmean-free-path ( $\operatorname{lmfp}$ ) regime. Thus, the reduction of neoclassical transport is one of the key issues for any future fusion reactor based on a non-axisymmetric configuration.

In this paper we study the neoclassical transport in strongly inward-shifted configurations of the LHD and find a neoclassical-transport-optimized configuration by evaluating a mono-energetic neoclassical transport coefficient. In order to compare the neoclassical transport properties of this optimized configuration with those of "advanced stellarators", an effective helical ripple, $\varepsilon_{\text {eff }}$, is also evaluated from the transport coefficients obtained. The neoclassical transport coefficient in the $1 / v$ regime is proportional to $\varepsilon_{\text {eff }}$ and the neoclassical transport in the $\operatorname{lmfp}$ regime can be easily estimated by the value of $\varepsilon_{\text {eff }}$.

We evaluate a mono-energetic local transport coefficient using DCOM (Diffusion COefficient calculator by Monte-carlo method)[2] in which test particle orbits are followed solving the equations of motion in Boozer coordinates and the transport coefficient is evaluated statistically from the mean square displacement of the particles.

Figure 1 shows the mono-energetic transport coefficient evaluated by DCOM code normalized by the plateau value of the equivalent circular tokamak, $D_{p}=(\pi / 16)\left(v^{3} / R \omega_{c}{ }^{3}\right)$, where $v, R, l$, and $\omega_{c}$ are the velocity, the major radius, the rotational transform, and the cyclotron frequency, respectively. With respect to $1 / v$ transport, the optimum configuration is found when the magnetic axis has a major radius of $3.53 \mathrm{~m}$. In this case, the effective helical ripple is very small, remaining below $2 \%$ inside $4 / 5$ of the plasma radius (Fig. 2). This indicates that a strong inward shift of the magnetic axis can optimized the neoclassical transport of the LHD configuration to a level typical of so-called"advanced stella-rators".

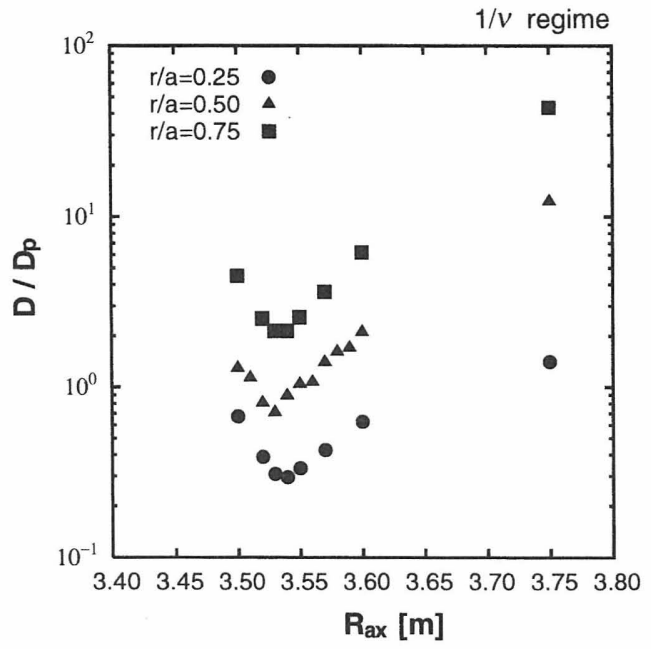

Fig. 1 Normalized neoclassical transport coefficients evaluated by DCOM as a function of the magnetic axis position in the $1 / v$ regime.

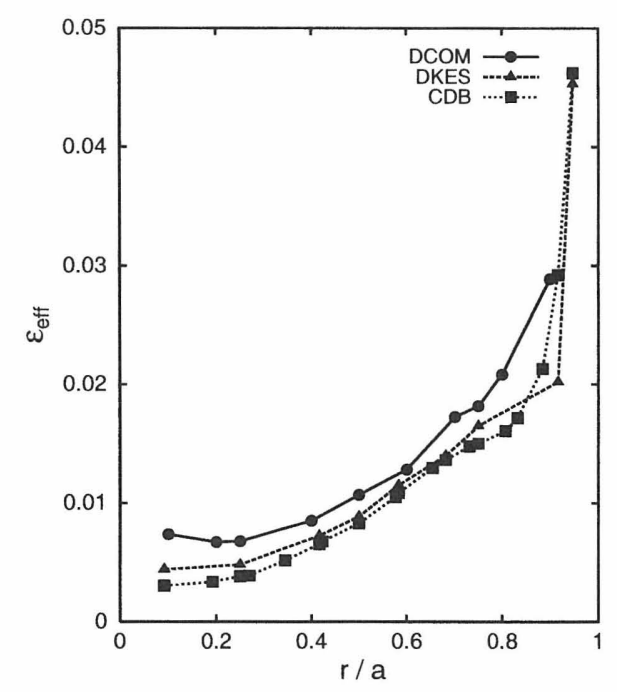

Fig. 2 Radial profiles of the effective helical ripple in the $R_{a x}=3.53 \mathrm{~m}$ configuration evaluated by DCOM, DKES and an analytic formula.

\section{REFERENCES}

[1] S. Murakami, et al, Nculear Fusion 42 (2002) in press.

[2] A. Wakasa, et al., J. Plasma Fusion Res. SERIES, Vol. 4 (2001) 408-412. 